OPEN ACCESS

Edited by:

Sengottayan Senthil-Nathan, Manonmaniam Sundaranar

University, India

Reviewed by:

Meghan Laturney,

University of California, Berkeley,

United States

Bimalendu B. Nath,

Savitribai Phule Pune University, India

*Correspondence:

Francesca Scolari

francesca.scolari@igm.cnr.it

Diana Pérez-Staples

diperez@uv.mx

Specialty section:

This article was submitted to

Invertebrate Physiology,

a section of the journal

Frontiers in Physiology

Received: 23 June 2021 Accepted: 14 September 2021

Published: 07 October 2021

Citation:

Scolari F, Khamis FM and

Pérez-Staples D (2021) Beyond

Sperm and Male Accessory Gland

Proteins: Exploring Insect

Reproductive Metabolomes.

Front. Physiol. 12:729440.

doi: 10.3389/fphys.2021.729440

\section{Beyond Sperm and Male Accessory Gland Proteins: Exploring Insect Reproductive Metabolomes}

\author{
Francesca Scolari ${ }^{1 *}$, Fathiya M. Khamis ${ }^{2}$ and Diana Pérez-Staples ${ }^{3 *}$ \\ ${ }^{1}$ Institute of Molecular Genetics (IGM)-CNR "Luigi Luca Cavalli-Sforza”, Pavia, Italy, ${ }^{2}$ International Centre of Insect Physiology \\ and Ecology (icipe), Nairobi, Kenya, ${ }^{3}$ Instituto de Biotecnología y Ecología Aplicada (INBIOTECA), Universidad Veracruzana, \\ Xalapa, Mexico
}

Insect seminal fluid, the non-sperm component of the ejaculate, comprises a variegated set of molecules, including, but not limited to, lipids, proteins, carbohydrates, salts, hormones, nucleic acids, and vitamins. The identity and functional role of seminal fluid proteins (SFPs) have been widely investigated, in multiple species. However, most of the other small molecules in insect ejaculates remain uncharacterized. Metabolomics is currently adopted to deepen our understanding of complex biological processes and in the last 15 years has been applied to answer different physiological questions. Technological advances in high-throughput methods for metabolite identification such as mass spectrometry and nuclear magnetic resonance (NMR) are now coupled to an expanded bioinformatics toolbox for large-scale data analysis. These improvements allow for the processing of smaller-sized samples and for the identification of hundreds to thousands of metabolites, not only in Drosophila melanogaster but also in disease vectors, animal, and agricultural pests. In this review, we provide an overview of the studies that adopted metabolomics-based approaches in insects, with a particular focus on the reproductive tract $(\mathrm{RT})$ of both sexes and the ejaculate. Progress in the field of metabolomics will contribute not only to achieve a deeper understanding of the composition of insect ejaculates and how they are affected by endogenous and exogenous factors, but also to provide increasingly powerful tools to decipher the identity and molecular interactions between males and females during and after mating.

Keywords: metabolomics, mating, ejaculate, seminal fluid, female post-mating response, mass spectrometry, nuclear magnetic resonance

\section{INTRODUCTION}

During mating, males are known to transfer to females not only their spermatozoa, but also non-sperm components in the ejaculate. The proteins within the seminal fluid (SF), regarded as the seminal fluid proteins (SFPs), have been widely characterized, initially in Drosophila melanogaster and later in several other insect species (see Meuti and Short, 2019 for a review). The non-protein fraction of the ejaculate includes hormones (e.g., 20E), nucleic, amino and fatty acids, lipids [e.g., the prostaglandins (PG) in crickets], carbohydrates, terpenoids, and defensive compounds (Poiani, 2006; Perry et al., 2013; Hopkins et al., 2018). Both SFPs and non-protein 
molecules have been shown to have a wide range of physiological and behavioral effects when interacting with female reproductive tissues (Avila et al., 2011), as compounds from both groups, including PGs and steroid hormones, are able to decrease sexual receptivity and stimulate oviposition in mated females (Stanley and Kim, 2011; Worthington et al., 2015). Although hundreds of SFPs have been identified, we are still limited in our understanding of the smaller ( $<1,500 \mathrm{Da}$; German et al., 2005) molecules of the non-protein fraction of the ejaculate that make up the male reproductive metabolome (RM). These compounds are likely involved in long-term sperm storage, sexual receptivity, and, ultimately, reproductive success and sexual conflict. Ejaculate proteins and the RM are likely interacting, as shown by their concerted antimicrobial role in conferring protection from diseases in the honeybee, Apis mellifera (Peng et al., 2016). The RM comprises molecules with various physical and chemical properties that can be delivered to females during mating bound to sperm or packaged into vesicles (Hopkins et al., 2017). This implies that different methods need to be adopted to identify and measure specific RM components, and the respective results have to be integrated.

Metabolomics, i.e., the comprehensive analysis of the metabolites in a system (Fiehn, 2002; Kamleh et al., 2009a), is a powerful tool to (i) identify and measure metabolites in insect organs and biological fluids (e.g., hemolymph and ejaculates), (ii) characterize the metabolic state of a tissue/ organ, and (iii) provide a global overview of the changes associated with insect physiology (e.g., development, diet, mating, and aging). Metabolomics is thus instrumental to provide information that complements the genomic, transcriptomic, and proteomic profile of the reproductive system and allows the identification of key molecules involved in metabolic pathways essential to insect reproduction and to other fundamental biological roles.

The aim of this mini-review is to provide a summary of published studies on metabolomics of insect reproductive tissues and ejaculates. We will describe the methodological features of metabolomics-based experiments, the different technological resources available as well as the findings achieved so far. Finally, we will focus on the future directions and challenges of this research field, both in terms of basic and applied science.

\section{TECHNICAL APPROACHES AND CHALLENGES OF INSECT METABOLOMICS}

Metabolomics-based approaches require the optimal integration of several different experimental and analytical components, namely (i) efficient sample collection to obtain a large number of replicates to cover the maximum possible variation, (ii) sample preparation protocols, (iii) chemical analyses, and (iv) data processing and bioinformatics.

Metabolomics studies can be performed through either targeted or untargeted approaches (Almontashiri et al., 2020). Targeted metabolomics studies focus on the identification of pre-defined chemically characterized compounds. This approach requires sample preparations optimized to select the physiochemical properties of the specific compounds of interest, followed by analysis with chromatography and/or mass spectrometry. This strategy relies on an a priori hypothesis and intrinsically identifies a limited set of metabolites. An untargeted metabolomics approach is usually adopted to trace a global profiling of all the metabolites present in a sample, producing a "metabolic fingerprint." To achieve this goal, it is essential to (1) integrate data from different metabolic profiling technologies with comprehensive chemical information from complex mixtures and (2) process the acquired data with powerful bioinformatic tools, annotate metabolites, perform statistical analyses, identify metabolic pathways, and integrate metabolomics with other omics data.

The first aspect of any accurate metabolomic study is the collection of the samples, which have to be processed and frozen in liquid nitrogen. A rapid and efficient sample homogenization in an organic solvent is essential to release the intracellular metabolites, precipitate the proteins, and terminate the metabolic reactions. Given that metabolite turnover is fast, the sample collection/processing needs to be optimized to stop/quench the enzymatic reactions immediately. The final metabolite measure has to take into account sample loss and different extract efficiencies. Frozen samples can be stored at $-80^{\circ} \mathrm{C}$, but a fast analysis is recommended to avoid instability of specific metabolites over time. Importantly, several rounds of analysis using different biological samples collected in independent experimental replicates should be carried out (Cox et al., 2017; Onyango et al., 2020). Metabolomics can be influenced by a range of pre-analytical factors (e.g., sample collection, aliquoting, transport, storage, and thawing) and standardizing these steps is essential particularly for field samples, for which handling time may be longer. Moreover, in the case of field samples, quantitative environmental data and molecular profiles should be associated with the biochemical properties revealed by metabolomics in a multi-omics and modeling frame to obtain more robust data (Nagler et al., 2018).

The two most common analytical platforms used in metabolomics studies are mass spectrometry (MS) analyzers (combined with gas or liquid chromatography - GC or LC, but also capillary electrophoresis, CE) and nuclear magnetic resonance (NMR) spectrometers (Soga et al., 2003; Kamleh et al., 2009a; Cox et al., 2017). NMR spectroscopy has been applied for the analysis of metabolic data since the 1970s (Wilson et al., 1974) and strongly contributed to launching the field of metabolomics (German et al., 2005; Wishart, 2008). MS and NMR are complementary due to their respective strengths and weaknesses, resulting in different sets of metabolites that can be detected. While NMR detects the most abundant metabolites in a sample, MS detects those that are readily ionizable (Bhinderwala et al., 2018). NMR-based techniques are robust, fast, reproducible, high-throughput, and non-destructive, but are less sensitive than MS, associated with a higher instrument cost, not optimal for targeted analyses, and allow the identification of a smaller set of metabolites. MS-based methods are associated with lower reproducibility, require more complex sample preparation and tissue extraction, 
and have longer analysis than NMR and higher costs (Amberg et al., 2017; Miggiels et al., 2019; Pinu et al., 2019).

The analysis of raw metabolomics data consists of the extraction of signals that correspond to individual chemicals and is organized in the following steps: (1) extraction of the chromatographic peaks, i.e., detection and extraction of peaks at specific $\mathrm{m} / \mathrm{z}$ values, within a defined mass tolerance, and with their retention time (RT); (2) RT alignment to facilitate sample comparison by adjusting RT shifts between injections; (3) deconvolution/componentization based on RT similarity and peak shape to reduce data complexity; and (4) data filtering to eliminate baseline noise. These steps are critically described in several articles (Worley and Powers, 2012; Saccenti et al., 2014; Yi et al., 2016; Cox et al., 2017), and their analysis goes beyond the scope of our review.

The final step in a metabolomics study is the interpretation of the produced data to determine whether observed changes in the metabolite profiles are associated with metabolic pathways. This can be achieved using different online databases, such as Metaboanalyst, The Kyoto Encyclopedia of Genes and Genomes (KEGG), and Reactome (see Cox et al., 2017 for a detailed review). Moreover, different mathematical and computational methods are available for integrating gene expression and metabolomics data (McIntyre et al., 2020; Jendoubi, 2021; Misra, 2021).

Untargeted metabolomics ("discovery metabolomics"), while holding the potential for finding new key biomarkers, has a drawback: the identification of chemicals with mass features not matching any compounds in the available databases, which may limit the discovery of specialized compounds playing key biological roles in target species (Al-Wathiqui et al., 2016). Indeed, de novo identification of a compound not having an accessible fragmentation pattern and not confidently identifiable by spectral library searches is particularly challenging and frequently regarded as one of the most relevant bottlenecks in metabolomics. Thus, several approaches have to be integrated, including elemental structure identification and mock fragmentations of candidate structures, with particular attention devoted to functional groups that are likely to fragment and undergo complete ionization (Gertsman and Barshop, 2018). Moreover, one of the biggest analytical challenges is to separate and identify very similar metabolites (e.g., isomeric and isobaric) with different biological functions. To overcome this issue and improve identification, strategies are being developed to verify retention behavior by updating retention information between different experiments allowing smaller tolerance for RT shifts, which decrease the power of compound databases (Opialla et al., 2020).

\section{INITIAL STUDIES OF INSECT METABOLOMES}

Nuclear magnetic resonance-based metabolomics had been initially applied in Drosophila species to trace metabolic profiles in hypoxic conditions (Feala et al., 2007) and to address questions such as those related to stress resistance
(Malmendal et al., 2006, 2013; Feala et al., 2007; Overgaard et al., 2007; Coquin et al., 2008; Pedersen et al., 2008; Cox et al., 2017), aging (Mishur and Rea, 2012; Kapranas et al., 2016), and the effects of pesticides and pollutants on insect biochemistry (Yan et al., 2018). The first applications of MS-based metabolomics in insects (Latocha et al., 2000; Ueyama et al., 2005; Fyrst et al., 2008) included the metabolomic profiling of mutants, including rosy (Kamleh et al., 2008, 2009b; Bratty et al., 2011).

In the last decade, GC-MS methods have been applied to address effects of stresses, including heat, freezing, and desiccation (Michaud and Denlinger, 2007; Robert Michaud et al., 2008; Koštál et al., 2011; Colinet et al., 2012; Mamai et al., 2014; Cox et al., 2017; Horvath et al., 2021). Nanospray ionization tandem mass spectrometry approaches have been used to investigate ammonia and nitrogen metabolism in Aedes aegypti (Scaraffia et al., 2006, 2008), as well as the changes in metabolomic profiles in response to different diets and after pathogen infection in Anopheles (Champion et al., 2017). A GC-TOF/MS system was employed to characterize the global metabolic changes induced by irradiation in the Japanese pine sawyer, Monochamus alternatus (Qu et al., 2014), showing the potential of metabolomics as a tool to acquire information on the mechanisms underlying radiation-induced sterility, with wide implications for pest control. In insects, metabolomics studies are exponentially increasing (Snart et al., 2015), particularly in the last 5 years.

\section{ADVANCED CROSS-PLATFORM DATA INTEGRATION}

Currently, the analytical challenges in metabolomics include the need to integrate data derived from more than one platform to produce more robust data and a wider metabolome coverage, maximizing the detection of possible metabolites in the target system (see Aliferis and Jabaji, 2011; Ćeranić et al., 2020; Roca et al., 2021 for reviews). Recent studies are showing that this integration is particularly powerful. For example, Zhou and colleagues combined UPLC-QTOF-MS and GC-Q-MS to explore the metabolic changes induced by azadirachtin, the main active component of neem-based pesticides, on the larvae of the tephritid fruit fly Bactrocera dorsalis (Zhou et al., 2020). Another recent study provided a comprehensive analysis of the changes in the whole-body metabolome of the tiger mosquito Aedes albopictus after infection with the Zika virus (Onyango et al., 2020) through integrating GC-TOF for identification of primary metabolites (e.g., carbohydrates, proteins, amino acids, vitamins, and hydroxyl acids), LC/Q-TOF CSH for lipid profiling, and HILIC LC/Q-TOF for analysis of biogenic amines (e.g., monoamine neurotransmitters and polyamines). The hot topic of the biochemical response to arbovirus infection and pathology in insect vectors recently included metabolomics as a powerful tool to be applied to both cell lines (Perera et al., 2012; Melo et al., 2016; Bottino-Rojas et al., 2019) and insect tissues (Chotiwan et al., 2018; Shrinet et al., 
2018). These studies are contributing to achieve a deeper understanding of the metabolic environments in mosquito tissues where viral infection, replication, and dissemination occur. These findings are essential to identify biochemical markers that could be targeted to interfere with arbovirus infection, thus expanding the toolbox for vector control. Similarly, studies on metabolic fingerprints between hosts and herbivores may lead to the development of new pesticides (Riach et al., 2019; Sanchez-Arcos et al., 2019).

\section{METABOLOMICS APPLIED TO THE CHARACTERIZATION OF INSECT REPRODUCTIVE METABOLITES}

While a majority of the first insect metabolomics studies were based on a "whole-organism homogenate" approach, thereafter the importance of tracing tissue-specific metabolomes began to emerge (Carvalho et al., 2012; Chintapalli et al., 2012, 2013). These seminal studies showed that the metabolomes of each tissue strongly differ and were also significantly divergent from the data obtained from whole-body analyses (Chintapalli et al., 2013). This finding opened new avenues to better understand physiological processes including diapause (Lu et al., 2014), as well as profiling the metabolites present in the hemolymph (Killiny et al., 2017). However, to date, only a handful of studies focusing on the metabolic composition of insect reproductive tissues are available (Table 1). Interestingly though, these studies are showing the feasibility of performing metabolomics analysis on small amounts of insect tissues and organs.

\section{Metabolomics of the Male and Female Reproductive Organs}

Studies tracing the metabolome of male reproductive organs play a key role in (1) determining the relative contribution of testes ( $\mathrm{T}$ ), male accessory glands (mAGs), and other secretory tissues in the production of ejaculate metabolites and (2) investigating phenomena such as oxidative stress and infertility, similarly to what has been done in mammals (Aitken and Baker, 2020; Ribas-Maynou and Yeste, 2020). Only two studies so far adopted a metabolomics approach to this purpose. The first was done in Drosophila, and testes, mAGs, and ovaries were included among the organs for which a metabolomic profile was traced (Chintapalli et al., 2013). The testes showed high levels of glutathione, which may provide protection against oxidative stress, and of phosphoarginine, carnitine, and acetyl carnitine, important for sperm motility. The lipid pattern in these organs revealed that phosphatidylethanolamine, phosphatidylcholine, and ether lipids are particularly abundant, reflecting the important role of lipids as sperm membrane components (Chintapalli et al., 2013). The mAGs metabolome showed uniquely high levels of methionine derivatives, such as S-adenosylmethionine, putatively involved in polyamine biosynthesis. The ovary metabolome suggested high metabolic activity, with pathways
TABLE 1 | Metabolomics studies performed on insect reproductive tissues and ejaculates.

\begin{tabular}{|c|c|c|c|c|}
\hline Species & $\begin{array}{l}\text { Organs/ } \\
\text { Ejaculate }\end{array}$ & $\begin{array}{l}\text { Metabolomics } \\
\text { platform }\end{array}$ & $\begin{array}{l}\text { Biological } \\
\text { question } \\
\text { addressed }\end{array}$ & Reference \\
\hline $\begin{array}{l}\text { Photinus } \\
\text { pyralis }\end{array}$ & Spermatophore & LC-HRAM-MS & $\begin{array}{l}\text { Characterize } \\
\text { synthesis, } \\
\text { composition, } \\
\text { and fate of the } \\
\text { spermatophore }\end{array}$ & $\begin{array}{l}\text { Al-Wathiqui } \\
\text { et al., } 2016\end{array}$ \\
\hline $\begin{array}{l}\text { Drosophila } \\
\text { melanogaster }\end{array}$ & $\begin{array}{l}\text { mAGs, testes, } \\
\text { and ovaries }\end{array}$ & $\begin{array}{l}\text { ZIC-HILIC } \\
\text { LC-Orbitrap } \\
\text { MS }\end{array}$ & $\begin{array}{l}\text { Explore the } \\
\text { metabolite } \\
\text { composition of } \\
\text { individual } \\
\text { tissues }\end{array}$ & $\begin{array}{l}\text { Chintapalli } \\
\text { et al., } 2013\end{array}$ \\
\hline Culex pipiens & mAGs & NMR & $\begin{array}{l}\text { Evaluate the } \\
\text { differences in } \\
\text { the } \\
\text { metabolome } \\
\text { among adult } \\
\text { males reared } \\
\text { on different } \\
\text { diets }\end{array}$ & $\begin{array}{l}\text { Huck et al., } \\
2021\end{array}$ \\
\hline Apis mellifera & Spermatheca & LC-MS & $\begin{array}{l}\text { Assess } \\
\text { changes in } \\
\text { spermathecal } \\
\text { metabolome } \\
\text { between virgin } \\
\text { and new-laying } \\
\text { queens }\end{array}$ & $\begin{array}{l}\text { Liu et al., } \\
2020\end{array}$ \\
\hline
\end{tabular}

mAGs, male accessory glands; LC-HRAM-MS, liquid chromatography hyphenated with high-resolution accurate mass spectrometry; ZIC-HILIC LC-Orbitrap MS, zwitterionichydrophilic interaction chromatography liquid chromatography-Orbitrap mass spectrometry; NMR, nuclear magnetic resonance; and LC-MS, liquid chromatographymass spectrometry.

related to antioxidant defense, stem cell formation, lipid and terpenoid biosynthesis, and energy reserve being particularly represented (Chintapalli et al., 2013).

The second study focused on the metabolomic analysis of Culex pipiens mAGs and revealed that the adult male diet had an impact on the composition of these organs, likely affecting SF composition (Huck et al., 2021). By integrating fecundity assays and metabolic results, the authors showed that nutritionally deprived males divert metabolic resources toward energy metabolism instead of reproduction. In particular, NMR spectroscopy allowed the identification of formic acid, which may enhance sperm motility, glucose, lactic acid, fatty acyls, and triglycerides, which were positively correlated with dietary sugar content (Huck et al., 2021). Interestingly, both glucose and lactic acid were detected in the male reproductive tissues of mammals (Mumcu et al., 2020), suggesting a functional conservation that deserves further exploration.

\section{Metabolomics of Insect Ejaculates}

So far, only one study focusing on the metabolomic characterization of an insect male ejaculate has been published. Specifically, this study aimed at characterizing the metabolome of the spermatophore in the common Eastern firefly Photinus pyralis (Al-Wathiqui 
et al., 2016). Spermatophores are sperm-containing capsules composed of secretions derived from the mAGs (Lewis et al., 2014) and also from the testes, as shown in the tsetse fly Glossina m. morsitans (Scolari et al., 2016). Al-Wathiqui et al. (2016) applied a multi-omics approach integrating MS/MS proteomics and liquid chromatography hyphenated with high-resolution accurate mass spectrometry (LC-HRAM-MS) metabolomics to characterize the spermatophore components in $P$. pyralis. In addition to 208 proteins, an untargeted metabolomics analysis found that several mass features were exclusively present (or present at significantly higher abundance) in the spermatophore, further supporting the idea that targeted and untargeted metabolomics are not mutually exclusive but can be profitably integrated. Given the absence of matches to any compounds in the KEGG database, some of these chemicals may be specialized molecules yet to be identified. Using a targeted metabolomics analysis, lucibufagin, a known firefly defensive toxin, was detected in both the spermatophore and the male body (Al-Wathiqui et al., 2016). Similarly, in the beetle Neopyrochroa flabellate, defensive terpenoids (cantharidin) have been found to be ingested by males, stored in the mAGs, and then transferred and used by females for egg defense (Eisner et al., 1996). Therefore, metabolomics will help us understand the evolution of ejaculate nuptial gifts that may protect females and/or eggs against predators or microbial attacks.

\section{Metabolomics of Female Sperm Storage Organs}

Analysis of the sperm storage organs holds great potential as it has implications for the rearing and conservation of beneficial insect species, such as honeybees, and in the optimization of strategies for the control of pest and vector populations in the wild. Indeed, by unravelling the physiological mechanisms underlying sperm storage, it may be possible to develop tools to affect fertility, as well as expand our knowledge on oogenesis. Furthermore, by comparing metabolites in reproductive tissues between unmated and mated females and males, it may be possible to discern among metabolites produced, received, stored, and potentially used by females. Similarly to males, females produce proteins in their reproductive tract (RT) that mediate inter-sex molecular interactions (Schnakenberg et al., 2011; Sun and Spradling, 2013). Expanding knowledge on the small molecule set produced in female RT will contribute to further understanding the interactions between sexes. To assess the post-mating metabolic changes occurring in the spermatheca of Apis mellifera, an LC-MS analysis was performed to compare the metabolites of virgin (VQS) and new-laying queens (NLQS; Liu et al., 2020). Of the total 7,745 metabolites identified, 861 were found to be differentially expressed, with 384 being significantly higher in their abundance in NLQS, and 477 significantly lower. Authors detected changes in lipids and lipid-like molecules during sperm storage in NLQS, such as fatty acyls, glycerophospholopids, prenol lipids, and sterol lipids. These data are particularly relevant as they highlight key target metabolites and biosynthetic pathways with potential important roles in sperm storage and reproductive success.

\section{APPLICATIONS OF INSECT METABOLOMICS: KNOWLEDGE GAPS AND FUTURE TRENDS IN REPRODUCTIVE BIOLOGY}

From a basic science perspective, the characterization of RMs will allow (i) identification of key players supporting sperm function, storage and use, and female fertility, (ii) evaluation of the impact of insect physiology and the environment on ejaculate composition, (iii) a better understanding of the respective contribution of specific male and female organs (Figure 1), and (iv) an expanded knowledge on how male-derived molecules interact with the female RT, particularly beyond SFPs. The RM will provide insights into ejaculate evolution, so that production costs of these complex molecules and fitness trade-offs can be determined and compared across species. Identification of non-protein components of the ejaculate, such as PGs, terpenes, and sexually transmitted pathogens is critical to understand how males and females may be under differential selection pressures on immune response, and regulation of remating rates to name a few.

Metabolomics may help in expansion of knowledge and management approaches for controlling agricultural and animal insect pests, as well as vectors of multiple diseases. From the applied point of view, metabolomics can contribute to the fingerprinting of the ejaculates produced by irradiated males in the context of the sterile insect technique (SIT; where the target species is mass-reared, sterilized, and released so males will mate with wild females transferring lethal dominant mutations in their sperm and thus avoiding offspring production; Dyck et al., 2021). This will allow for a novel and sophisticated male quality control and, more specifically, for the identification of markers of infertility, similar to what has been achieved in mammals (Zhao et al., 2018; Engel et al., 2019; Menezes et al., 2019; Murgia et al., 2020), to determine SF fertilizing capacity. The availability of these markers may help in identifying the metabolic pathways producing such key metabolites and will also enable identification of related gene candidate(s) that can be manipulated to either (i) explore strategies alternative to irradiation to achieve sterility, (ii) find pathways that could be targeted in the female to induce mortality after mating with manipulated males, and/or (iii) increase the fecundity and fertility of mass-reared insects.

Metabolomics is particularly promising also to investigate the metabolites produced by the gut microbiota that may have an impact on the quality of mass-reared insects, in relation to different types of larval and adult diets. This exciting area of research may lead to the identification of compounds to be exploited as probiotic supplements in larval diets and deciphering the functions of insect host-bacterial symbiont interactions. Bing et al. (2017) explored the metabolic co-evolution between G. m. morsitans and its obligate endosymbiont Wigglesworthia using a combined transcriptomics and metabolomics approach. The absence of the symbiont-derived $B$ vitamins affects tsetse fly metabolism, physiology, and survival in multiple ways and supports the key role of this symbiont in ensuring the fitness of the fly host. Although metabolomics studies are increasingly being undertaken, this field is ripe for future studies combining transcriptomics, proteomics, and metabolomics. 


\section{The Insect Reproductive Metabolome (RM)}
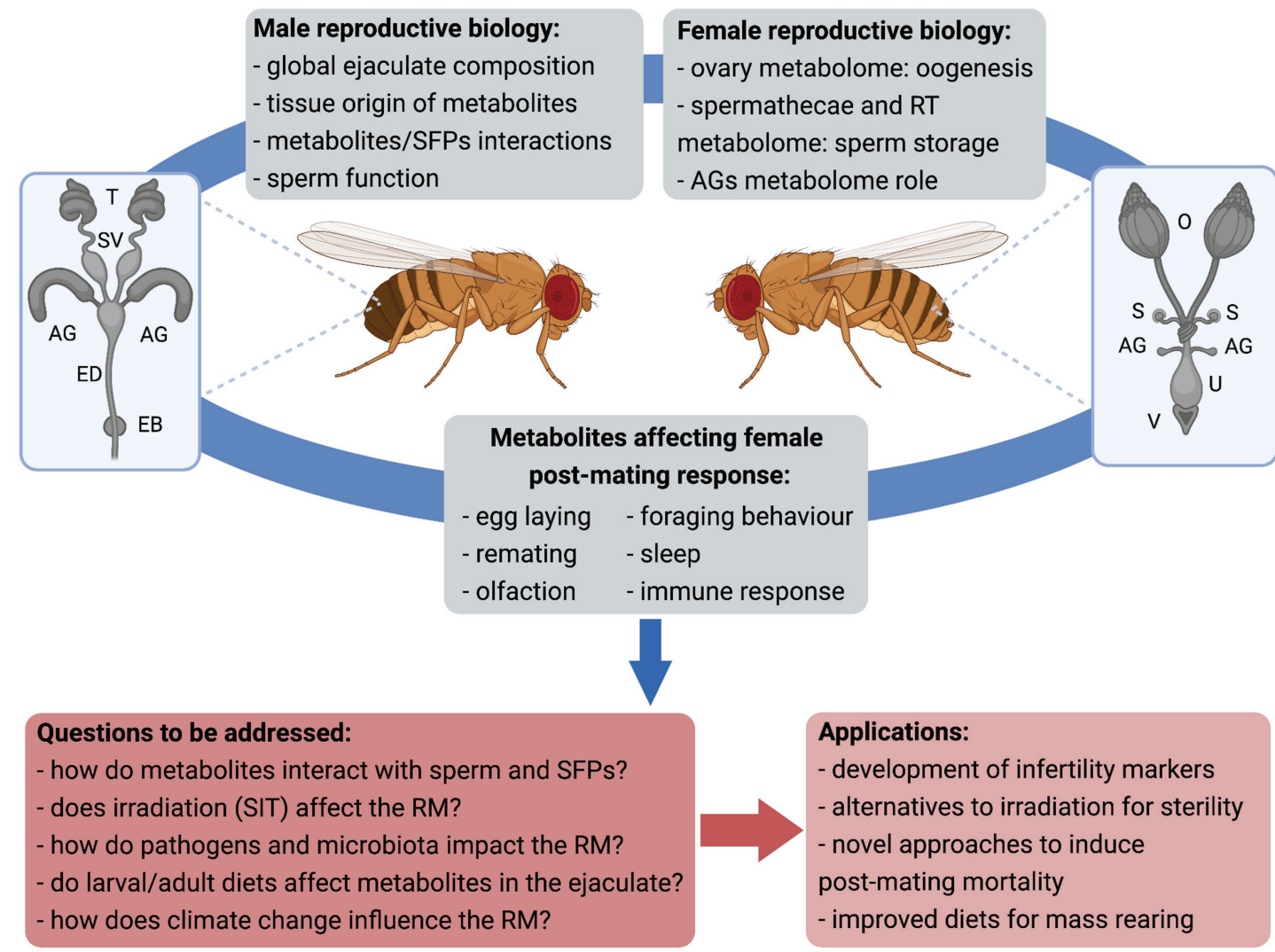

FIGURE 1 | The insect reproductive metabolome (RM). Metabolomics can be applied to the study of male and female reproductive biology and expand knowledge on the physiological mechanisms underlying inter-sexual interactions upon mating, with relevant practical application for insect control. SFPs, seminal fluid proteins; RT, reproductive tract; AG, accessory glands; T, testes; SV, seminal vesicles; ED, ejaculatory duct; EB, ejaculatory bulb; O, ovaries; S, spermathecae; U, uterus; V, vulva; SIT, sterile insect technique; and RM, reproductive metabolome. Figure created with BioRender.com.

Such an integrated approach can now be applied to studies comparing pre- and post-mating changes in females and males. Moreover, the integration of metabolomics with up-to-date metagenomics analyses can elucidate the role of symbionts in insect ejaculates. Indeed, insect ejaculate is not "sterile," but it is known to be populated by microbes and viruses (Poiani, 2006; Otti, 2015; Rowe et al., 2020). Several metabolic pathways triggered by mating and/or by the symbionts present in the male ejaculate may be revealed, thus opening novel research horizons.

\section{REFERENCES}

Aitken, R. J., and Baker, M. A. (2020). The role of genetics and oxidative stress in the etiology of male infertility-a unifying hypothesis? Front. Endocrinol. 11:581838. doi: 10.3389/fendo.2020.581838

Aliferis, K. A., and Jabaji, S. (2011). Metabolomics-a robust bioanalytical approach for the discovery of the modes-of-action of pesticides: a review. Pestic. Biochem. Physiol. 100, 105-117. doi: 10.1016/j.pestbp.2011.03.004

Almontashiri, N. A. M., Zha, L., Young, K., Law, T., Kellogg, M. D., Bodamer, O. A., et al. (2020). Clinical validation of targeted and untargeted metabolomics testing for genetic disorders: a 3 year comparative study. Sci. Rep. 10:9382. doi: $10.1038 / \mathrm{s} 41598-020-66401-2$

\section{AUTHOR CONTRIBUTIONS}

FS conceived the topic for the mini-review and prepared the figure. FS, DP-S, and FMK wrote the manuscript. All authors contributed to the article and approved the submitted version.

\section{ACKNOWLEDGMENTS}

The authors thank their families for their support during the writing of this mini-review.

Al-Wathiqui, N., Fallon, T. R., South, A., Weng, J. K., and Lewis, S. M. (2016) Molecular characterization of firefly nuptial gifts: a multi-omics approach sheds light on postcopulatory sexual selection. Sci. Rep. 6:38556. doi: 10.1038/srep38556

Amberg, A., Riefke, B., Schlotterbeck, G., Ross, A., Senn, H., Dieterle, F., et al. (2017). NMR and MS methods for metabolomics. Methods Mol. Biol. 1641, 229-258. doi: 10.1007/978-1-4939-7172-5_13

Avila, F. W., Sirot, L. K., LaFlamme, B. A., Rubinstein, C. D., and Wolfner, M. F. (2011). Insect seminal fluid proteins: identification and function. Annu. Rev. Entomol. 56, 21-40. doi: 10.1146/annurev-ento-120709-144823

Bhinderwala, F., Wase, N., DiRusso, C., and Powers, R. (2018). Combining mass spectrometry and NMR improves metabolite detection and annotation. J. Proteome Res. 17, 4017-4022. doi: 10.1021/acs.jproteome.8b00567 
Bing, X., Attardo, G. M., Vigneron, A., Aksoy, E., Scolari, F., Malacrida, A., et al. (2017). Unravelling the relationship between the tsetse fly and its obligate symbiont Wigglesworthia: transcriptomic and metabolomic landscapes reveal highly integrated physiological networks. Proc. R. Soc. B Biol. Sci. 284:20170360. doi: 10.1098/rspb.2017.0360

Bottino-Rojas, V., Pereira, L. O. R. R., Silva, G., Talyuli, O. A. C. C., Dunkov, B. C., Oliveira, P. L., et al. (2019). Non-canonical transcriptional regulation of heme oxygenase in Aedes aegypti. Sci. Rep. 9:13726. doi: 10.1038/ s41598-019-49396-3

Bratty, M. A., Hobani, Y., Dow, J. A. T., and Watson, D. G. (2011). Metabolomic profiling of the effects of allopurinol on Drosophila melanogaster. Metabolomics 7, 542-548. doi: 10.1007/s11306-011-0275-6

Carvalho, M., Sampaio, J. L., Palm, W., Brankatschk, M., Eaton, S., and Shevchenko, A. (2012). Effects of diet and development on the drosophila lipidome. Mol. Syst. Biol. 8:600. doi: 10.1038/msb.2012.29

Ćeranić, A., Bueschl, C., Doppler, M., Parich, A., Xu, K., Lemmens, M., et al. (2020). Enhanced metabolome coverage and evaluation of matrix effects by the use of experimental-condition-matched 13C-labeled biological samples in isotope-assisted LC-HRMS metabolomics. Meta 10:434. doi: 10.3390/ metabo10110434

Champion, C. J., Kukutla, P., Glennon, E. K. K., Wang, B., Luckhart, S., and $\mathrm{Xu}, \mathrm{J}$. (2017). Anopheles gambiae: metabolomic profiles in sugar-fed, bloodfed, and plasmodium falciparum-infected midgut. Dataset Pap. Sci. 2017, 1-49. doi: $10.1155 / 2017 / 8091749$

Chintapalli, V. R., Al Bratty, M., Korzekwa, D., Watson, D. G., and Dow, J. A. T. (2013). Mapping an atlas of tissue-specific Drosophila melanogaster metabolomes by high resolution mass spectrometry. PLoS One 8:e78066. doi: 10.1371/ journal.pone. 0078066

Chintapalli, V. R., Terhzaz, S., Wang, J., Al Bratty, M., Watson, D. G., Herzyk, P., et al. (2012). Functional correlates of positional and gender-specific renal asymmetry in drosophila. PLoS One 7:e32577. doi: 10.1371/journal.pone.0032577

Chotiwan, N., Andre, B. G., Sanchez-Vargas, I., Islam, M. N., Grabowski, J. M., Hopf-Jannasch, A., et al. (2018). Dynamic remodeling of lipids coincides with dengue virus replication in the midgut of Aedes aegypti mosquitoes. PLoS Pathog. 14:1006853. doi: 10.1371/journal.ppat.1006853

Colinet, H., Larvor, V., Laparie, M., and Renault, D. (2012). Exploring the plastic response to cold acclimation through metabolomics. Funct. Ecol. 26, 711-722. doi: 10.1111/j.1365-2435.2012.01985.x

Coquin, L., Feala, J. D., McCulloch, A. D., and Paternostro, G. (2008). Metabolomic and flux-balance analysis of age-related decline of hypoxia tolerance in drosophila muscle tissue. Mol. Syst. Biol. 4:233. doi: 10.1038/msb.2008.71

Cox, J. E., Thummel, C. S., and Tennessen, J. M. (2017). Metabolomic studies in drosophila. Genetics 206, 1169-1185. doi: 10.1534/genetics.117.200014

Dyck, V. A., Hendrichs, J., and Robinson, A. S. (2021). Sterile Insect Technique. New York: CRC Press.

Eisner, T., Smedley, S. R., Young, D. K., Eisner, M., Roach, B., and Meinwald, J. (1996). Chemical basis of courtship in a beetle (Neopyrochroa flabellata): Cantharidin as "nuptial gift". Proc. Natl. Acad. Sci. 93, 6499-6503. doi: 10.1073/pnas.93.13.6499

Engel, K. M., Baumann, S., Rolle-Kampczyk, U., Schiller, J., von Bergen, M., and Grunewald, S. (2019). Metabolomic profiling reveals correlations between spermiogram parameters and the metabolites present in human spermatozoa and seminal plasma. PLoS One 14:e0211679. doi: 10.1371/journal.pone.0211679

Feala, J. D., Coquin, L., McCulloch, A. D., and Paternostro, G. (2007). Flexibility in energy metabolism supports hypoxia tolerance in drosophila flight muscle: metabolomic and computational systems analysis. Mol. Syst. Biol. 3:99. doi: $10.1038 / \mathrm{msb} 4100139$

Fiehn, O. (2002). Metabolomics-the link between genotypes and phenotypes. Plant Mol. Biol. 48, 155-171. doi: 10.1023/A:1013713905833

Fyrst, H., Zhang, X., Herr, D. R., Hoe, S. B., Bittman, R., Phan, V. H., et al. (2008). Identification and characterization by electrospray mass spectrometry of endogenous drosophila sphingadienes. J. Lipid Res. 49, 597-606. doi: 10.1194/jlr.M700414-JLR200

German, J. B., Hammock, B. D., and Watkins, S. M. (2005). Metabolomics: building on a century of biochemistry to guide human health. Metabolomics 1, 3-9. doi: 10.1007/s11306-005-1102-8

Gertsman, I., and Barshop, B. A. (2018). Promises and pitfalls of untargeted metabolomics. J. Inherit. Metab. Dis. 41, 355-366. doi: 10.1007/ s10545-017-0130-7
Hopkins, B. R., Avila, F. W., and Wolfner, M. F. (2018). "Insect male reproductive glands and their products," in Encyclopedia of Reproduction. ed. M. K. Skinner (London: Elsevier), 137-144.

Hopkins, B. R., Sepil, I., and Wigby, S. (2017). Seminal fluid. Curr. Biol. 27, R404-R405. doi: 10.1016/j.cub.2017.03.063

Horvath, T. D., Dagan, S., and Scaraffia, P. Y. (2021). Unraveling mosquito metabolism with mass spectrometry-based metabolomics. Trends Parasitol. 37, 747-761. doi: 10.1016/j.pt.2021.03.010

Huck, D. T., Klein, M. S., and Meuti, M. E. (2021). Determining the effects of nutrition on the reproductive physiology of male mosquitoes. J. Insect Physiol. 129:104191. doi: 10.1016/j.jinsphys.2021.104191

Jendoubi, T. (2021). Approaches to integrating metabolomics and multi-omics data: a primer. Meta 11:184. doi: 10.3390/metabo11030184

Kamleh, M. A., Dow, J. A. T., and Watson, D. G. (2009a). Applications of mass spectrometry in metabolomic studies of animal model and invertebrate systems. Brief. Funct. Genom. Proteomics 8, 28-48. doi: 10.1093/bfgp/eln052

Kamleh, M. A., Hobani, Y., Dow, J. A. T., and Watson, D. G. (2008). Metabolomic profiling of drosophila using liquid chromatography Fourier transform mass spectrometry. FEBS Lett. 582, 2916-2922. doi: 10.1016/j.febslet.2008.07.029

Kamleh, M. A., Hobani, Y., Dow, J. A. T., Zheng, L., and Watson, D. G. (2009b). Towards a platform for the metabonomic profiling of different strains of Drosophila melanogaster using liquid chromatography-Fourier transform mass spectrometry. FEBS J. 276, 6798-6809. doi: 10.1111/j.1742-4658.2009.07397.x

Kapranas, A., Snart, C. J. P., Williams, H., Hardy, I. C. W., and Barrett, D. A. (2016). Metabolomics of aging assessed in individual parasitoid wasps. Sci. Rep. 6:34848. doi: 10.1038/srep34848

Killiny, N., Hijaz, F., El-Shesheny, I., Alfaress, S., Jones, S. E., and Rogers, M. E. (2017). Metabolomic analyses of the haemolymph of the Asian citrus psyllid Diaphorina citri, the vector of huanglongbing. Physiol. Entomol. 42, 134-145. doi: $10.1111 /$ phen.12183

Koštál, V., Korbelová, J., Rozsypal, J., Zahradníčková, H., Cimlová, J., Tomčala, A., et al. (2011). Long-term cold acclimation extends survival time at $0^{\circ} \mathrm{C}$ and modifies the metabolomic profiles of the larvae of the fruit fly Drosophila melanogaster. PLoS One 6:e25025. doi: 10.1371/journal.pone.0025025

Latocha, M., Chodurek, E., Kurkiewicz, S., Świątkowska, L., and Wilczok, T. (2000). Pyrolytic GC-MS analysis of melanin from black, gray and yellow strains of Drosophila melanogaster. J. Anal. Appl. Pyrolysis 56, 89-98. doi: 10.1016/S0165-2370(00)00082-6

Lewis, S. M., Vahed, K., Koene, J. M., Engqvist, L., Bussière, L. F., Perry, J. C., et al. (2014). Emerging issues in the evolution of animal nuptial gifts. Biol. Lett. 10:20140336. doi: 10.1098/rsbl.2014.0336

Liu, Z., Liu, F., Li, G., Chi, X., Wang, Y., Wang, H., et al. (2020). Metabolite support of long-term storage of sperm in the spermatheca of honeybee (Apis mellifera) queens. Front. Physiol. 11:574856. doi: 10.3389/ fphys.2020.574856

Lu, Y. X., Zhang, Q., and Xu, W. H. (2014). Global metabolomic analyses of the hemolymph and brain during the initiation, maintenance, and termination of pupal diapause in the cotton bollworm, Helicoverpa armigera. PLoS One 9:e99948. doi: 10.1371/journal.pone.0099948

Malmendal, A., Overgaard, J., Bundy, J. G., Sørensen, J. G., Nielsen, N. C., Loeschcke, V., et al. (2006). Metabolomic profiling of heat stress: hardening and recovery of homeostasis in drosophila. Am. J. Phys. Regul. Integr. Comp. Phys. 291, R205-R212. doi: 10.1152/ajpregu.00867.2005

Malmendal, A., Sørensen, J. G., Overgaard, J., Holmstrup, M., Nielsen, N. C., and Loeschcke, V. (2013). Metabolomic analysis of the selection response of Drosophila melanogaster to environmental stress: are there links to gene expression and phenotypic traits? Naturwissenschaften 100, 417-427. doi: 10.1007/s00114-013-1040-7

Mamai, W., Mouline, K., Blais, C., Larvor, V., Dabiré, K. R., Ouedraogo, G. A., et al. (2014). Metabolomic and ecdysteroid variations in Anopheles gambiae s.l. mosquitoes exposed to the stressful conditions of the dry season in Burkina Faso, West Africa. Physiol. Biochem. Zool. 87, 486-497. doi: $10.1086 / 675697$

McIntyre, L. M., Huertas, F., Moskalenko, O., Llansola, M., Felipo, V., Morse, A. M., et al. (2020). GAIT-GM: galaxy tools for modeling metabolite changes as a function of gene expression. bioRxiv [Preprint]. doi: 10.1101/2020.12.25.424407

Melo, C. F. O. R., de Oliveira, D. N., De Oliveira Lima, E., Guerreiro, T. M., Esteves, C. Z., Beck, R. M., et al. (2016). A lipidomics approach in the 
characterization of Zika-infected mosquito cells: potential targets for breaking the transmission cycle. PLoS One 11:e0164377. doi: 10.1371/journal. pone. 0164377

Menezes, E. B., Velho, A. L. C., Santos, F., Dinh, T., Kaya, A., Topper, E., et al. (2019). Uncovering sperm metabolome to discover biomarkers for bull fertility. BMC Genomics 20:714. doi: 10.1186/s12864-019-6074-6

Meuti, M. E., and Short, S. M. (2019). Physiological and environmental factors affecting the composition of the ejaculate in mosquitoes and other insects. Insects 10:74. doi: 10.3390/insects10030074

Michaud, M. R., and Denlinger, D. L. (2007). Shifts in the carbohydrate, polyol, and amino acid pools during rapid cold-hardening and diapauseassociated cold-hardening in flesh flies (Sarcophaga crassipalpis): a metabolomic comparison. J. Comp. Physiol. B. 177, 753-763. doi: 10.1007/ s00360-007-0172-5

Miggiels, P., Wouters, B., van Westen, G. J. P., Dubbelman, A.-C., and Hankemeier, T. (2019). Novel technologies for metabolomics: more for less. TrAC Trends Anal. Chem. 120:115323. doi: 10.1016/j.trac.2018.11.021

Mishur, R. J., and Rea, S. L. (2012). Applications of mass spectrometry to metabolomics and metabonomics: detection of biomarkers of aging and of age-related diseases. Mass Spectrom. Rev. 31, 70-95. doi: 10.1002/mas.20338

Misra, B. B. (2021). New software tools, databases, and resources in metabolomics: updates from 2020. Metabolomics 17:49. doi: 10.1007/s11306-021-01796-1

Mumcu, A., Karaer, A., Dogan, B., and Tuncay, G. (2020). Metabolomics analysis of seminal plasma in patients with idiopathic oligoasthenoteratozoospermia using high-resolution NMR spectroscopy. Andrology 8, 450-456. doi: 10.1111/ andr. 12707

Murgia, F., Corda, V., Serrenti, M., Usai, V., Santoru, M. L., Hurt, K. J., et al. (2020). Seminal fluid metabolomic markers of oligozoospermic infertility in humans. Meta 10:64. doi: 10.3390/metabo10020064

Nagler, M., Nägele, T., Gilli, C., Fragner, L., Korte, A., Platzer, A., et al. (2018). Eco-metabolomics and metabolic modeling: making the leap from model systems in the lab to native populations in the field. Front. Plant Sci. 9:1556. doi: $10.3389 /$ fpls.2018.01556

Onyango, M. G., Attardo, G. M., Kelly, E. T., Bialosuknia, S. M., Stout, J., Banker, E., et al. (2020). Zika virus infection results in biochemical changes associated with RNA editing, inflammatory and antiviral responses in Aedes albopictus. Front. Microbiol. 11:559035. doi: 10.3389/fmicb.2020.559035

Opialla, T., Kempa, S., and Pietzke, M. (2020). Towards a more reliable identification of isomeric metabolites using pattern guided retention validation. Meta 10:457. doi: 10.3390/metabo10110457

Otti, O. (2015). Genitalia-associated microbes in insects. Insect Sci. 22, 325-339. doi: $10.1111 / 1744-7917.12183$

Overgaard, J., Malmendal, A., Sørensen, J. G., Bundy, J. G., Loeschcke, V., Nielsen, N. C., et al. (2007). Metabolomic profiling of rapid cold hardening and cold shock in Drosophila melanogaster. J. Insect Physiol. 53, 1218-1232. doi: 10.1016/j.jinsphys.2007.06.012

Pedersen, K. S., Kristensen, T. N., Loeschcke, V., Petersen, B. O., Duus, J. Ø., Nielsen, N. C., et al. (2008). Metabolomic signatures of inbreeding at benign and stressful temperatures in Drosophila melanogaster. Genetics 180, 1233-1243. doi: 10.1534/genetics.108.089144

Peng, Y., Grassl, J., Millar, A. H., and Baer, B. (2016). Seminal fluid of honeybees contains multiple mechanisms to combat infections of the sexually transmitted pathogen Nosema apis. Proc. R. Soc. B Biol. Sci. 283:20151785. doi: 10.1098/ rspb.2015.1785

Perera, R., Riley, C., Isaac, G., Hopf-Jannasch, A. S., Moore, R. J., Weitz, K. W., et al. (2012). Dengue virus infection perturbs lipid homeostasis in infected mosquito cells. PLoS Pathog. 8:e1002584. doi: 10.1371/journal.ppat.1002584

Perry, J. C., Sirot, L., and Wigby, S. (2013). The seminal symphony: how to compose an ejaculate. Trends Ecol. Evol. 28, 414-422. doi: 10.1016/j. tree.2013.03.005

Pinu, F. R., Goldansaz, S. A., and Jaine, J. (2019). Translational metabolomics: current challenges and future opportunities. Meta 9:108. doi: 10.3390/ metabo9060108

Poiani, A. (2006). Complexity of seminal fluid: a review. Behav. Ecol. Sociobiol. 60, 289-310. doi: 10.1007/s00265-006-0178-0

Qu, L. J., Wang, L. J., Zhang, Y. A., Wang, Q. H., Wang, Y. Z., Zhao, T. H., et al. (2014). Radiation-induced metabolomic changes in sterile male Monochamus alternatus (Coleoptera: Cerambycidae). J. Insect Sci. 14:166. doi: $10.1093 /$ jisesa/ieu028
Riach, A. C., Perera, M. V. L., Florance, H. V., Robinson, L. A., Penfield, S. D., and Hill, J. K. (2019). Metabolic fingerprints reveal how an insect metabolome is affected by different larval host plant species. Arthropod Plant Interact. 13, 571-579. doi: 10.1007/s11829-019-09671-6

Ribas-Maynou, J., and Yeste, M. (2020). Oxidative stress in male infertility: causes, effects in assisted reproductive techniques, and protective support of antioxidants. Biology 9:77. doi: 10.3390/biology9040077

Robert Michaud, M., Benoit, J. B., Lopez-Martinez, G., Elnitsky, M. A., Lee, R. E., and Denlinger, D. L. (2008). Metabolomics reveals unique and shared metabolic changes in response to heat shock, freezing and desiccation in the Antarctic midge, Belgica Antarctica. J. Insect Physiol. 54, 645-655. doi: 10.1016/j.jinsphys.2008.01.003

Roca, M., Alcoriza, M. I., Garcia-Cañaveras, J. C., and Lahoz, A. (2021). Reviewing the metabolome coverage provided by LC-MS: focus on sample preparation and chromatography-A tutorial. Anal. Chim. Acta 1147, 38-55. doi: $10.1016 /$ j.aca.2020.12.025

Rowe, M., Veerus, L., Trosvik, P., Buckling, A., and Pizzari, T. (2020). The reproductive microbiome: an emerging driver of sexual selection, sexual conflict, mating systems, and reproductive isolation. Trends Ecol. Evol. 35, 220-234. doi: 10.1016/j.tree.2019.11.004

Saccenti, E., Hoefsloot, H. C. J., Smilde, A. K., Westerhuis, J. A., and Hendriks, M. M. W. B. (2014). Reflections on univariate and multivariate analysis of metabolomics data. Metabolomics 10, 361-374. doi: 10.1007/s11306-013-0598-6

Sanchez-Arcos, C., Kai, M., Svatoš, A., Gershenzon, J., and Kunert, G. (2019). Untargeted metabolomics approach reveals differences in host plant chemistry before and after infestation with different pea aphid host races. Front. Plant Sci. 10:188. doi: 10.3389/fpls.2019.00188

Scaraffia, P. Y., Tan, G., Isoe, J., Wysocki, V. H., Wells, M. A., and Miesfeld, R. L. (2008). Discovery of an alternate metabolic pathway for urea synthesis in adult Aedes aegypti mosquitoes. Proc. Natl. Acad. Sci. U. S. A. 105, 518-523. doi: $10.1073 /$ pnas. 0708098105

Scaraffia, P. Y., Zhang, Q., Wysocki, V. H., Isoe, J., and Wells, M. A. (2006). Analysis of whole body ammonia metabolism in Aedes aegypti using [15N]labeled compounds and mass spectrometry. Insect Biochem. Mol. Biol. 36, 614-622. doi: 10.1016/j.ibmb.2006.05.003

Schnakenberg, S. L., Matias, W. R., and Siegal, M. L. (2011). Sperm-storage defects and live birth in drosophila females lacking spermathecal secretory cells. PLoS Biol. 9:e1001192. doi: 10.1371/journal.pbio.1001192

Scolari, F., Benoit, J. B., Michalkova, V., Aksoy, E., Takac, P., Abd-Alla, A. M. M., et al. (2016). The spermatophore in Glossina morsitans morsitans: insights into male contributions to reproduction. Sci. Rep. 6:20334. doi: 10.1038/srep20334

Shrinet, J., Bhavesh, N. S., and Sunil, S. (2018). Understanding oxidative stress in Aedes during chikungunya and dengue virus infections using integromics analysis. Viruses 10:314. doi: 10.3390/v10060314

Snart, C. J. P., Hardy, I. C. W., and Barrett, D. A. (2015). Entometabolomics: applications of modern analytical techniques to insect studies. Entomol. Exp. Appl. 155, 1-17. doi: 10.1111/eea.12281

Soga, T., Ohashi, Y., Ueno, Y., Naraoka, H., Tomita, M., and Nishioka, T. (2003). Quantitative metabolome analysis using capillary electrophoresis mass spectrometry. J. Proteome Res. 2, 488-494. doi: 10.1021/pr034020m

Stanley, D., and Kim, Y. (2011). Prostaglandins and their receptors in insect biology. Front. Endocrinol. 2:105. doi: 10.3389/fendo.2011.00105

Sun, J., and Spradling, A. C. (2013). Ovulation in drosophila is controlled by secretory cells of the female reproductive tract. eLife 2:e00415. doi: 10.7554/eLife.00415

Ueyama, M., Chertemps, T., Labeur, C., and Wicker-Thomas, C. (2005). Mutations in the desat 1 gene reduces the production of courtship stimulatory pheromones through a marked effect on fatty acids in Drosophila melanogaster. Insect Biochem. Mol. Biol. 35, 911-920. doi: 10.1016/j.ibmb.2005.03.007

Wilson, D. M., Burlingame, A. L., Cronholm, T., and Sjövall, J. (1974). Deuterium and carbon-13 tracer studies of ethanol metabolism in the rat by $2 \mathrm{H}$, 1H-decoupled 13C nuclear magnetic resonance. Biochem. Biophys. Res. Commun. 56, 828-835. doi: 10.1016/0006-291X(74)90680-9

Wishart, D. S. (2008). Quantitative metabolomics using NMR. Trends Anal. Chem. 27, 228-237. doi: 10.1016/j.trac.2007.12.001

Worley, B., and Powers, R. (2012). Multivariate analysis in metabolomics. Curr. Metabolomics 1, 92-107. doi: 10.2174/2213235X11301010092

Worthington, A., Jurenka, R., and Kelly, C. (2015). Mating for malederived prostaglandin: a functional explanation for the increased fecundity of mated female crickets? J. Exp. Biol. 218, 2720-2727. doi: 10.1242/jeb.121327 
Yan, P.-C., Wen, C.-W., Zhang, S.-Z., Zhang, Z.-D., Xu, J.-P., and Deng, M.-J. (2018). A toxicological, metabonomic and transcriptional analysis to investigate the property of mulberry 1-deoxynojirimycin against the growth of Samia cynthia ricini. Pestic. Biochem. Physiol. 152, 45-54. doi: 10.1016/j. pestbp.2018.08.009

Yi, L., Dong, N., Yun, Y., Deng, B., Ren, D., Liu, S., et al. (2016). Chemometric methods in data processing of mass spectrometry-based metabolomics: a review. Anal. Chim. Acta 914, 17-34. doi: 10.1016/j.aca.2016.02.001

Zhao, K., Zhang, J., Xu, Z., Xu, Y., Xu, A., Chen, W., et al. (2018). Metabolomic profiling of human spermatozoa in idiopathic asthenozoospermia patients using gas chromatography-mass spectrometry. Biomed. Res. Int. 2018:8327506. doi: 10.1155/2018/8327506

Zhou, Y., Qin, Q., Zhang, P. W., Chen, X. T., Liu, B. J., Cheng, D. M., et al. (2020). Integrated LC-MS and GC-MS-based untargeted metabolomics studies of the effect of azadirachtin on Bactrocera dorsalis larvae. Sci. Rep. 10:2306. doi: $10.1038 /$ s41598-020-58796-9
Conflict of Interest: The authors declare that the research was conducted in the absence of any commercial or financial relationships that could be construed as a potential conflict of interest.

Publisher's Note: All claims expressed in this article are solely those of the authors and do not necessarily represent those of their affiliated organizations, or those of the publisher, the editors and the reviewers. Any product that may be evaluated in this article, or claim that may be made by its manufacturer, is not guaranteed or endorsed by the publisher.

Copyright (c) 2021 Scolari, Khamis and Pérez-Staples. This is an open-access article distributed under the terms of the Creative Commons Attribution License (CC BY). The use, distribution or reproduction in other forums is permitted, provided the original author(s) and the copyright owner(s) are credited and that the original publication in this journal is cited, in accordance with accepted academic practice. No use, distribution or reproduction is permitted which does not comply with these terms. 